\title{
Interval type-2 fuzzy adaptive strategy for fault tolerant control based on new faulty model design: Application to DSIM under broken rotor bars fault
}

\author{
Noureddine Layadi ${ }^{1 *}$, Samir Zeghlache ${ }^{1}$, Ali Djerioui ${ }^{1}$, Hemza Mekki $^{1}$, Azeddine Houari ${ }^{2}$, Mohamed-Fouad Benkhoris ${ }^{2}$, Fouad \\ Berrabah $^{3}$ \\ ${ }^{1}$ Laboratoire de Génie Electrique, Department of Electrical Engineering, Faculty of Technology, University Mohamed Boudiaf \\ of M'Sila, BP 166, Ichbilia 28000, Algeria \\ ${ }^{2}$ IREENA Laboratory, University of Nantes, Saint-Nazaire, France \\ ${ }^{3}$ Department of Electrical Engineering, Faculty of Technology, University Mohamed Boudiaf of M'Sila, BP 166, Ichbilia 28000, \\ Algeria
}

Corresponding Author Email: layadinoureddine1@gmail.com

https://doi.org/10.18280/mmc_a.910407

Received: 12 August 2018

Accepted: 30 November 2018

\section{Keywords:}

double star induction machine, interval type-

2 fuzzy logic system, adaptive control, sliding mode control, fault tolerant control, broken rotor bars

\begin{abstract}
This paper presents a fault tolerant control (FTC) based on the type-2 fuzzy logic system (IT2FLS) using an adaptive control law for a double star induction machine (DSIM) under broken rotor bars (BRB) fault of a squirrel-cage in order to improve its reliability and availability. The adaptive fuzzy control is designed to compensate for the fault effect. The proposed FTC is able to maintain acceptable performance in the event of BRB. The stability of the closed-loop is verified by exploitation of Lyapunov theory. To proof the performance and effectiveness of the proposed FTC, a comparative study within sliding mode control (SMC) is carried out. Obtained results show that the proposed FTC has a better robustness against the BRB fault.
\end{abstract}

\section{INTRODUCTION}

The double star induction machine (DSIM) belongs to the category of multiphase induction machines (MIM). It has been selected as the best choice because of its many advantages over its three-phase counterpart. The DSIM has been proposed for different fields of industry that need high power such as electric hybrid vehicles, locomotive traction, ship propulsion and many other applications where the safety condition is required such as aerospace and offshore wind energy systems. DISM not only guarantees a decrease of rotor harmonics currents and torque pulsations but it also has many other advantages such as: reliability, power segmentation and higher efficiency. DSIM has a greater fault tolerance; it can continue to operate and maintain rotating flux even with open-phase faults thanks to the greater number of degrees of freedom that it owns compared to the three-phase machines [1]

The motors installed in the industry are $85 \%$ of squirrel cage motors [2]. Induction motors are subject to various faults; about $40 \%$ to $50 \%$ are bearing faults, $5 \%$ to $10 \%$ are severe rotor faults, and $30 \%$ to $40 \%$ are stator-related faults [3]. Broken bars fault has proved dangerous and may be the cause of other faults in the stator and the rotor itself because a broken rotor bar considerably increases the currents flowing in the neighboring bars, which causes the increase of the mechanical stresses (constraints) and consequently causes the rupture of the corresponding bars [4]. BRB fault can be caused by failures in the rotor fabrication process, overloads (mechanical stress), mechanical cracks or thermal stress [5].

Compared to stator faults, detection of the broken rotor bar especially at an early stage is quite difficult, the appearance of this type of fault does not cause the machine to stop immediately but reduces its performance, for this reason, many diagnoses in the literature are used to detect the broken bar fault in squirrel cage rotors of induction motors [6], these detection processes exploit the information provided by the main signals of the machine such as: motor currents, torque, instantaneous power (active and reactive), mechanical vibrations and flux. Despite its drawbacks to the voltage source in case of voltage harmonic distortion, the MCSA method is widely used to detect a broken rotor bar [7]. The MCSA is largely used as a test in the process of monitoring induction machines, especially in the detection of electrical and mechanical faults due to its simplicity and efficiency. The MCSA does not need additional sensors; it is based solely on the analysis of the reliable information provided by the stator currents during start-up or in steady state [8].

Inspired by the research of Belhamdi, this paper proposes a fuzzy type-2 adaptive control for DSIM with defective rotor in order to compensate the fault effect after estimating uncertainties. The modeling of the BRB fault in the (d-q) reference frame with consideration of homopolar components is carried out for the first time on this kind of multiphase machine. The method of modeling faults in this paper allows the development of other more innovative control techniques in the future. The proposed FTC is tested in healthy and defective conditions with other control methods applied recently on a six-phase induction machine $[9,10]$. Performance of these controllers is investigated and compared in terms of tracking reference of rotor speed, electromagnetic torque and rotor flux. This paper has made several contributions in relation to the recent research concerning the FTC:

A novel adaptive fuzzy controller to master correctly the torque, flux and speed tracking of a DSIM with BRB fault has been proposed, in this contribution, the application of the adaptive fuzzy control as FTC for DSIM in a faulty case is 
performed for the first time.

Unlike, the adaptive control law has been implemented in all steps, which increases the tolerance of the controller, in addition, the proposed FTC treated a defective machine while was treating a healthy doubly-fed induction motor (DFIM) [11-12].

Compared to the work of Mekki, the proposed FTC does not need an observer or internal model to compensate the fault effect.

In Masumpoor [13], an adaptive sliding-mode type-2 neurofuzzy control of an induction motor is proposed, in order to compensate parametric uncertainties and disturbances, the parameters of this fusion of control are adjusted thanks to an online learning algorithm based on sliding-mode training algorithm and type-2 fuzzy systems adaptive controller, the simulation results provide a robust tracking of reference but remain insufficient because the system global stability that is needed to validate this control strategy is not discussed. In this paper the stability analysis of the closed-loop system is strictly proven.

Compared with González-PrietoI, and Mahmoud [14-15], where authors present an FTC of six-phase induction motor under open-circuit fault and an FTC of five-phase induction machine under open gate transistor faults, respectively, the degree of severity of the fault dealt with in this paper is more important since open phase fault tolerance is a specific feature of multiphase machines thanks to the high number of phases.

The remainder of this paper is organized as follows; the next section describes the DSIM faulty model. The design of an interval type-2 fuzzy logic adaptive controller for DSIM is carried out in section 3. Simulation results and their discussions are given in section 4 . The last section is reserved for conclusion.

\section{DSIM FAULTY MODEL}

In order to have a light model, we consider the rotor as a balanced three-phase system. In Figure 1, the squirrel cage rotor is replaced by an equivalent three phase windings (single star winding) with equivalent resistance $R_{r}$ and leakage $L_{r}$. When the rotor of the DSIM is broken, the rotor resistance is different from the nominal value [16], therefore, to simulate a $\mathrm{BRB}$ in the double star induction machine; we increase the resistance of a rotor phase by adding a defective resistance " $e$ ".

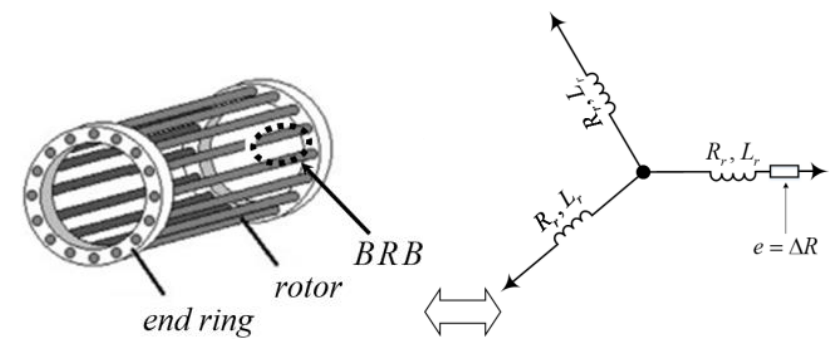

Figure 1. Simulation of BRB in DSIM

The first-order differential equations of the rotor voltages in the DSIM can be presented in the natural "abc" reference frame as:

$\left[V_{r}^{a b c}\right]=\left[R_{r}\right]\left[I_{r}^{a b c}\right]+\frac{d}{d t}\left[\Phi_{r}^{a b c}\right]$
With:

$$
\begin{aligned}
& {\left[R_{r}\right]=\left[\begin{array}{ccc}
R_{r} & 0 & 0 \\
0 & R_{r} & 0 \\
0 & 0 & R_{r}
\end{array}\right] ;} \\
& {\left[\Phi_{r}^{a b c}\right]=\left[\begin{array}{lll}
\varphi_{r a} & \varphi_{r b} & \varphi_{r c}
\end{array}\right]^{T}} \\
& {\left[I_{r}^{a b c}\right]=\left[\begin{array}{lll}
i_{r a} & i_{r b} & i_{r c}
\end{array}\right]^{T}} \\
& {\left[V_{r}^{a b c}\right]=\left[\begin{array}{lll}
v_{r a} & v_{r b} & v_{r c}
\end{array}\right]^{T}}
\end{aligned}
$$

where: $\left[R_{r}\right]$ is the matrix of resistances, $\left[\Phi_{r}^{a b c}\right]$ is the flux vector, $\left[I_{r}^{a b c}\right]$ is the currents vector and $\left[V_{r}^{a b c}\right]$ is the voltages vector.

When BRB fault occurs, the resistances matrix becomes as follow:

$$
\left[R_{r}^{B R B}\right]=\left[\begin{array}{ccc}
R_{r} & 0 & 0 \\
0 & R_{r} & 0 \\
0 & 0 & R_{r}+e
\end{array}\right]
$$

In this case, the voltage equation in (1) becomes:

$$
\left[V_{r}^{a b c}\right]=\left[R_{r}^{B R B}\right]\left[I_{r}^{a b c}\right]+\frac{d}{d t}\left[\Phi_{r}^{a b c}\right]
$$

By applying the park transformation that conserves energy on (2), we obtain the voltages equations in the (d-q) reference frame:

$\left[V_{r}^{d q o}\right]=\left[P_{r}(\theta)\right]\left[R_{r}^{B R B}\right]\left[P_{r}(\theta)\right]^{-1}\left[I_{r}^{d q o}\right]+$

$\left[P_{r}(\theta)\right] \frac{d}{d t}\left\{\left[P_{r}(\theta)\right]^{-1}\right\}\left[\Phi_{r}^{d q o}\right]+\frac{d}{d t}\left[\Phi_{r}^{d q o}\right]$

Where: $\left[V_{r}^{d q o}\right]=\left[\begin{array}{lll}v_{r d} & v_{r q} & v_{r o}\end{array}\right]^{T}$ is the voltages vector, $\left[I_{r}^{d q o}\right]=\left[\begin{array}{lll}i_{r d} & i_{r q} & i_{r o}\end{array}\right]^{T}$ is the currents vector and $\left[\Phi_{r}^{d q o}\right]=$ $\left[\begin{array}{lll}\varphi_{r d} & \varphi_{r q} & \varphi_{r o}\end{array}\right]^{T}$ is the rotor flux vector. by:

$\left[P_{r}(\theta)\right]$ is the rotor winding transformation matrix, is given

$$
\begin{aligned}
& {\left[P_{r}(\theta)\right]=} \\
& \sqrt{\frac{2}{3}}\left[\begin{array}{ccc}
\cos \left(\theta_{s}-\theta_{r}\right) & \cos \left(\theta_{s}-\theta_{r}-\frac{2 \pi}{3}\right) & \cos \left(\theta_{s}-\theta_{r}+\frac{2 \pi}{3}\right) \\
-\sin \left(\theta_{s}-\theta_{r}\right) & -\sin \left(\theta_{s}-\theta_{r}-\frac{2 \pi}{3}\right) & -\sin \left(\theta_{s}-\theta_{r}+\frac{2 \pi}{3}\right) \\
\frac{1}{\sqrt{2}} & \frac{1}{\sqrt{2}} & \frac{1}{\sqrt{2}}
\end{array}\right] \\
& \text { With: }\left\{\begin{array}{l}
\omega_{r}=\frac{d}{d t} \theta_{r} \\
\omega_{s}=\frac{d}{d t} \theta_{s} .
\end{array}\right.
\end{aligned}
$$

Finally, The DSIM model in presence of BRB faults are given by the following equations:

$$
\begin{aligned}
& \frac{d}{d t} \Omega=\frac{1}{J}\left[p^{2} \frac{L_{m}}{L_{m}+L_{r}} \varphi_{r}\left(i_{s q 1}+i_{s q 2}\right)-p T_{L}-K_{f} \Omega\right] \\
& \frac{d}{d t} \varphi_{r}=\frac{-R_{r}}{L_{r}+L_{m}} \varphi_{r}+\frac{L_{m} R_{r}}{L_{r}+L_{m}}\left(i_{s d 1}+i_{s d 2}\right)+\Gamma_{1} \\
& \frac{d}{d t} i_{s d 1}=\frac{1}{L_{s 1}}\left\{v_{s d 1}-R_{s 1} i_{s d 1}+\omega_{s}\left(L_{s 1} i_{s q 1}+T_{r} \varphi_{r} \omega_{g l}\right)\right\}+\Gamma_{2} \\
& \frac{d}{d t} i_{s q 1}=\frac{1}{L_{s 1}}\left\{v_{s q 1}-R_{s 1} i_{s q 1}-\omega_{s}\left(L_{s 1} i_{s d 1}+\varphi_{r}\right)\right\}+\Gamma_{3} \\
& \frac{d}{d t} i_{s o 1}=\frac{1}{L_{s 1}}\left(v_{s o 1}-R_{s 1} i_{s o 1}\right) \\
& \frac{d}{d t} i_{s d 2}=\frac{1}{L_{s 2}}\left\{v_{s d 2}-R_{s 2} i_{s d 2}+\omega_{s}\left(L_{s 2} i_{s q 2}+T_{r} \varphi_{r} \omega_{g l}\right)\right\}+\Gamma_{4}
\end{aligned}
$$


$\frac{d}{d t} i_{s q 2}=\frac{1}{L_{s 2}}\left\{v_{s q 2}-R_{s 2} i_{s q 2}-\omega_{s}\left(L_{s 2} i_{s d 2}+\varphi_{r}\right)\right\}+\Gamma_{5}$

$\frac{d}{d t} i_{s o 2}=\frac{1}{L_{s 2}}\left(v_{s o 2}-R_{s 2} i_{s o 2}\right)$

where $\Gamma_{i} \quad i=\overline{1,5}$ represent the fault terms due to broken bar fault, they are given by:

$$
\Gamma_{1}=-\left(\frac{R_{r}}{L_{r}+L_{m}} \frac{\alpha}{\beta\left(L_{r}+L_{m}\right)}-\frac{\omega_{g l}}{\beta}\right) \varphi_{r}+\left(\frac{\alpha}{\beta} \frac{L_{m}}{L_{r}+L_{m}}-\right.
$$

$\left.\frac{R_{r} L_{m}}{L_{r}+L_{m}}\right)\left(i_{s d 1}+i_{s d 2}\right)$;

$$
\begin{aligned}
& \Gamma_{2}=\left(\frac{L_{r}}{\eta}-T_{r}\right) \frac{\omega_{s} \omega_{g l} \varphi_{r}}{L_{s 2}} \\
& \Gamma_{3}=-\frac{L_{r} \gamma}{L_{s 2}} \omega_{s} \omega_{g l} \varphi_{r} ; \\
& \Gamma_{4}=\frac{1}{L_{s 2}}\left(\frac{L_{r}}{\eta}-T_{r}\right) \omega_{s} \omega_{g l} \varphi_{r} ; \\
& \Gamma_{5}=-\frac{L_{r}}{L_{s 2} \gamma} \omega_{s} \omega_{g l} \varphi_{r} .
\end{aligned}
$$

With:

$\eta=-\frac{a_{6} a_{2}^{2}-2 a_{2} a_{3} a_{5}+a_{4} a_{3}^{2}+a_{1} a_{5}^{2}-a_{1} a_{4} a_{6}}{-a_{3}^{2}+a_{1} a_{6}} ;$

$\boldsymbol{\mu}=-\frac{a_{6} a_{2}^{2}-2 a_{2} a_{3} a_{5}+a_{4} a_{3}^{2}+a_{1} a_{5}^{2}-a_{1} a_{4} a_{6}}{a_{2} a_{3}-a_{1} a_{5}} ;$

$\gamma=\frac{a_{2}\left(a_{6} a_{2}-2 a_{3} a_{5}\right)+a_{4} a_{3}^{2}+a_{1}\left(a_{5}^{2}-a_{4} a_{6}\right)}{a_{2} a_{6}-a_{3} a_{5}} ;$

$\alpha=\frac{a_{2}+\left(a_{3}-\frac{a_{1} a_{5}}{a_{2}}\right)\left(a_{5}-\frac{a_{3} a_{4}}{a_{2}}\right)}{\left(a_{6}-\frac{a_{3} a_{5}}{a_{2}}\right)-\frac{a_{1} a_{4}}{a_{2}}} ;$

$\beta=\frac{a_{4}}{a_{2}}+\frac{a_{5}\left(a_{5}-\frac{a_{4} a_{3}}{a_{2}}\right)}{a_{2}\left(a_{6}-\frac{a_{5} a_{3}}{a_{2}}\right)}$

Where:

$a_{1}=\frac{e}{3}+r r-\frac{e}{6} \cos \left(2 \theta_{r}-2 \theta_{s}\right)-\frac{\sqrt{3}}{6} e \sin \left(2 \theta_{r}-2 \theta_{s}\right)$;

$a_{2}=\frac{e}{3} \cos \left(2 \theta_{r}-2 \theta_{s}+\frac{\pi}{6}\right)$;

$a_{3}=-\frac{\sqrt{2}}{3} e \cos \left(\theta_{r}-\theta_{s}+\frac{\pi}{3}\right)$;

$a_{4}=\frac{e}{3}+r r+\frac{e}{6} \cos \left(2 \theta_{r}-2 \theta_{s}\right)+\frac{\sqrt{3}}{6} e \sin \left(2 \theta_{r}-2 \theta_{s}\right)$;

$a_{5}=-\frac{\sqrt{2}}{3} e \cos \left(\theta_{s}-\theta_{r}+\frac{\pi}{6}\right)$;

$a_{6}=\frac{e}{3}+r r$.

\section{DESIGN OF AN INTERVAL TYPE-2 FUZZY LOGIC ADAPTIVE CONTROLLER FOR DSIM}

The objective is to design an adaptive fuzzy control scheme for an uncertain DSIM model in the presence of BRB faults to properly manage flux and speed tracking. The role of the type2 fuzzy systems is to approximate the local nonlinearities of each subsystem, while the fuzzy parameters are adjusted in real time by adaptive laws, respecting the stability and convergence of the system according to the Lyapunov theory until the desired tracking performance is reached. To easily design the proposed controller, we operate with the DSIM faulty model developed in (5)-(12), in the presence of BRB faults, we have:

$$
\begin{aligned}
& \frac{d}{d t} \Omega=\frac{p^{2}}{J} \frac{L_{m}}{L_{m}+L_{r}} \varphi_{r}\left(i_{s q 1}+i_{s q 2}\right)+f_{1} \\
& \frac{d}{d t} \varphi_{r}=\frac{L_{m} R_{r}}{L_{r}+L_{m}}\left(i_{s d 1}+i_{s d 2}\right)+f_{2} \\
& \frac{d}{d t} i_{s d 1}=\frac{1}{L_{s 1}} v_{s d 1}+f_{3} \\
& \frac{d}{d t} i_{s q 1}=\frac{1}{L_{s 1}} v_{s q 1}+f_{4} \\
& \frac{d}{d t} i_{s o 1}=\frac{1}{L_{s 1}} v_{s o 1}+f_{5}
\end{aligned}
$$

$$
\begin{aligned}
& \frac{d}{d t} i_{s d 2}=\frac{1}{L_{s 2}} v_{s d 2}+f_{6} \\
& \frac{d}{d t} i_{s q 2}=\frac{1}{L_{s 2}} v_{s q 2}+f_{7} \\
& \frac{d}{d t} i_{s o 2}=\frac{1}{L_{s 2}} v_{s o 2}+f_{8}
\end{aligned}
$$

where:

$$
\begin{aligned}
& f_{1}=-\frac{p}{J} T_{L}-\frac{K_{f}}{J} \Omega ; \\
& f_{2}=\frac{-R_{r}}{L_{r}+L_{m}} \varphi_{r}+\Gamma_{1} ; \\
& f_{3}=\frac{-R_{s 1}}{L_{s 1}} i_{s d 1}+\omega_{s} i_{s q 1}+\frac{\omega_{s} T_{r} \varphi_{r} \omega_{g l}}{L_{s 1}}+\Gamma_{2} ; \\
& f_{4}=\frac{-R_{s 1}}{L_{s 1}} i_{s q 1}-\omega_{s} i_{s d 1}-\frac{\omega_{s} \varphi_{r}}{L_{s 1}}+\Gamma_{3} ; \\
& f_{5}=\frac{-R_{s 1}}{L_{s 1}} i_{s o 1} ; \\
& f_{6}=\frac{-R_{s 2}}{L_{s 2}} i_{s d 2}+\omega_{s} i_{s q 2}+\frac{\omega_{s} T_{r} \varphi_{r} \omega_{g l}}{L_{s 2}}+\Gamma_{4} ; \\
& f_{7}=\frac{-R_{s 2}}{L_{s 2}} i_{s q 2}-\omega_{s} i_{s d 2}-\frac{\omega_{s} \varphi_{r}}{L_{s 2}}+\Gamma_{5} ; \\
& f_{8}=\frac{-R_{s 2}}{L_{s 2}} i_{s o 2} .
\end{aligned}
$$

The nonlinear functions $h_{i}\left(\bar{x}_{i}\right) i=\overline{1,8}$ can be estimated by the IT2FLS as follows:

$\hat{h}_{i}\left(\bar{x}_{i}\right)=\theta_{i}^{T} \psi_{i}\left(\bar{x}_{i}\right), i=1, \ldots, 8$

where: $\bar{x}_{i}$ is the input vector, $\theta_{i}$ is the adjusted vector parameter and $\psi_{i}\left(\bar{x}_{i}\right)$ is the average of the basic functions calculated using IT2FLS (each basic function is computed as the average of the corresponding left and right basic functions).

Let us define:

The actual functions $h_{i}\left(\bar{x}_{i}\right)$ are given by:

$h_{i}\left(\bar{x}_{i}\right)=\theta_{i}^{* T_{i}\left(\overline{\bar{x}}_{l}\right)_{i}\left(\bar{x}_{i}\right)} i=\overline{1,8}$

The use of the optimal parameters $\theta_{i}^{*}$ is only for analytical purposes. For this reason, integrating the controller does not require its value [11]. The parametric errors are given by:

$\tilde{\theta}_{i}=\theta_{i}^{*}-\theta_{i}, i=1, \ldots, 8$

$\bar{\omega}_{i}\left(\bar{x}_{i}\right)$ are the approximation errors, such as: $\left|\bar{\omega}_{i}\left(\bar{x}_{i}\right)\right| \leq \overline{\bar{\omega}}_{i}$ Where $\overline{\bar{\omega}}_{i}$ are the unknown positive parameters. In order to achieve precise flux and speed tracking, some assumptions have been put:

Assumption1. The nonlinear functions $f_{i}\left(\bar{x}_{i}\right) i=\overline{1,8}$ are continuous nonlinear functions assumed to be unknown.

Assumption2. The reference signals $\Omega^{*}, \varphi_{r}^{*}, i_{s d 1}^{*}, i_{s q 1}^{*}, i_{s d 2}^{*}$ $i_{s q 2}^{*}, i_{s o 1}^{*}, i_{s o 2}^{*}$ and their first derivatives are bounded and continuous.

Assumption3. Rotor and stator currents and rotor speed are available for measurement. The tracking errors and their filtered errors are given by:

For rotor speed

$\tilde{\Omega}(t)=\Omega(t)-\Omega^{*}, S_{\Omega}=\tilde{\Omega}(t)+\lambda_{\Omega} \int_{0}^{t} \tilde{\Omega}(\tau) d \tau$ with $\tilde{\Omega}(0)=$ 0

For rotor flux

$\tilde{\varphi}_{r}(t)=\varphi_{r}(t)-\varphi_{r}^{*}, S_{\varphi}=\tilde{\varphi}_{r}(t)+\lambda_{\varphi} \int_{0}^{t} \tilde{\varphi}_{r}(\tau) d \tau$, with

$\tilde{\varphi}_{r}(0)=0$ 
For stator currents

$\tilde{l}_{s d 1}(t)=i_{s d 1}(t)-i_{s d 1}^{*}, S_{i s d 1}=\tilde{l}_{s d 1}(t)+\lambda_{i s d 1} \int_{0}^{t} \tilde{l}_{s d 1}(\tau) d \tau$, with $\tilde{l}_{s d 1}(0)=0$

$\tilde{l}_{s d 2}(t)=i_{s d 2}(t)-i_{s d 2}^{*}, S_{i s d 2}=\tilde{l}_{s d 2}(t)+\lambda_{i s d 2} \int_{0}^{t} \tilde{l}_{s d 2}(\tau) d \tau$, with $\tilde{l}_{s d 2}(0)=0$

$\tilde{l}_{s q 1}(t)=i_{s q 1}(t)-i_{s q 1}^{*}, S_{i s q 1}=\tilde{l}_{s q 1}(t)+\lambda_{i s q 1} \int_{0}^{t} \tilde{l}_{s q 1}(\tau) d \tau$, with $\tilde{i}_{s q 1}(0)=0$

$\tilde{l}_{s q 2}(t)=i_{s q 2}(t)-i_{s q 2}^{*}, S_{i s q 2}=\tilde{l}_{s q 2}(t)+\lambda_{i s q 2} \int_{0}^{t} \tilde{l}_{s q 2}(\tau) d \tau$, with $\tilde{l}_{s q 2}(0)=0$

For homopolar components

$\tilde{l}_{s o 1}(t)=i_{s o 1}(t)-i_{s o 1}^{*}, S_{i s o 1}=\tilde{l}_{s o 1}(t)+\lambda_{i s o 1} \int_{0}^{t} \tilde{l}_{s o 1}(\tau) d \tau$, with $\tilde{l}_{s o 1}(0)=0$

$\tilde{l}_{s o 2}(t)=i_{s o 2}(t)-i_{\text {So } 2}^{*}, S_{i s o 2}=\tilde{l}_{s o 2}(t)+\lambda_{\text {iso } 2} \int_{0}^{t} \tilde{l}_{s o 2}(\tau) d \tau$, with $\tilde{l}_{\text {so } 2}(0)=0$

where: $\lambda_{\Omega}, \lambda_{\varphi_{r}}, \lambda_{i s d 1}, \lambda_{i s d 2}, \lambda_{i s q 1}, \lambda_{i s q 2}, \lambda_{i s o 1}$ and $\lambda_{i s o 2}$ are strictly positive design parameters, and we admit that:

$\left\{\begin{array}{l}i_{s q 1}+i_{s q 2}=i_{s q}, \quad i_{s d 1}+i_{s d 2}=i_{s d} \\ i_{s q 1}^{*}=i_{s q 2}^{*}=\frac{i_{s q}^{*}}{2}, \quad i_{s d 1}^{*}=i_{s d 2}^{*}=\frac{i_{s d}^{*}}{2} \\ i_{s o 1}^{*}=0, \quad i_{s o 2}^{*}=0\end{array}\right.$

The following adaptive fuzzy control laws are made in the case where the dynamics of DSIM is uncertain:

$i_{s q}^{*}=\frac{J\left(L_{m}+L_{r}\right)}{p^{2} L_{m}}\left(-\theta_{1}^{T} \psi_{1}\left(\bar{x}_{1}\right)-k_{11} S_{\Omega}-k_{12} \tanh \left(\frac{s_{\Omega}}{\varepsilon_{i s q}}\right)\right)$

$i_{s d}^{*}=\frac{L_{r}+L_{m}}{L_{m} R_{r}}\left(-\theta_{2}^{T} \psi_{2}\left(\bar{x}_{2}\right)-k_{21} S_{\varphi_{r}}-k_{22} \tanh \left(\frac{S_{\varphi_{r}}}{\varepsilon_{i s d}}\right)\right)$

$v_{s d 1}=L_{s 1}\left(-\theta_{3}^{T} \psi_{3}\left(\bar{x}_{3}\right)-k_{31} S_{i s d 1}-k_{32} \tanh \left(\frac{s_{i s d 1}}{\varepsilon_{i s d 1}}\right)\right)$

$v_{s q 1}=L_{s 1}\left(-\theta_{4}^{T} \psi_{4}\left(\bar{x}_{4}\right)-k_{41} S_{i s q 1}-k_{42} \tanh \left(\frac{s_{i s q 1}}{\varepsilon_{i s q 1}}\right)\right)$

$v_{s o 1}=-\theta_{5}^{T} \psi_{5}\left(\bar{x}_{5}\right)-k_{51} S_{i s o 1}-k_{52} \tanh \left(\frac{s_{i s o 1}}{\varepsilon_{i s o 1}}\right)$

$v_{s d 2}=L_{s 2}\left(-\theta_{6}^{T} \psi_{6}\left(\bar{x}_{6}\right)-k_{61} S_{i s d 2}-k_{62} \tanh \left(\frac{s_{i s d 2}}{\varepsilon_{i s d 2}}\right)\right)$

$v_{s q 2}=L_{s 2}\left(-\theta_{7}^{T} \psi_{7}\left(\bar{x}_{7}\right)-k_{71} S_{i s q 1}-k_{72} \tanh \left(\frac{s_{i s q 2}}{\varepsilon_{i s q 2}}\right)\right)$

$v_{s o 2}=-\theta_{8}^{T} \psi_{8}\left(\bar{x}_{8}\right)-k_{81} S_{i s o 2}-k_{82} \tanh \left(\frac{s_{i s o 2}}{\varepsilon_{i s o 2}}\right)$

Where: the design parameters $k_{i 1}$ remain constants for $i=$ $\overline{1,8} . \varepsilon_{i s q}, \varepsilon_{i s d}, \varepsilon_{i s d 1}, \varepsilon_{i s q 1}, \varepsilon_{i s o 1}, \varepsilon_{i s d 2}, \varepsilon_{i s q 2}$ and $\varepsilon_{i s o 2}$ are absolutely positive design constants, usually are small. $\bar{\mu}_{\tilde{F}_{k}^{i}}\left(x_{k}\right)$ is the abbreviation hyperbolic tangent function. The interval type-2 fuzzy system has as inputs, the following vectors: $\bar{x}_{1}=$
$\left[\Omega^{*}, \Omega\right]^{T}, \bar{x}_{2}=\left[\varphi_{r}^{*}, \varphi_{r}\right]^{T}, \bar{x}_{3}=\left[i_{s d 1}, i_{s q 1}\right]^{T}, \bar{x}_{4}=\left[i_{s q 1}, i_{s d 1}\right]^{T}$, $\bar{x}_{5}=\left[i_{s o 1}^{*}, i_{s o 1}\right]^{T}, \bar{x}_{6}=\left[i_{s d 2}, i_{s q 2}\right]^{T}, \bar{x}_{7}=\left[i_{s q 2}, i_{s d 2}\right]^{T}, \bar{x}_{8}=$ $\left[i_{s o 2}^{*}, i_{s o 2}\right]^{T}$. According to [11]: to estimate the unknown fuzzy vectors $\left(\theta_{i}^{*}\right)$ and the unknown parameters $\left(k_{i 2}^{*}\right)$ for $i=\overline{1,8}$, we adopt the following adaptive laws:

$\dot{\theta}_{1}=-\sigma_{\theta_{1}} \gamma_{\theta_{1}} \theta_{1}+\gamma_{\theta_{1}} S_{\Omega} \psi_{1}\left(\bar{x}_{1}\right)$

$\dot{\theta}_{2}=-\sigma_{\theta_{2}} \gamma_{\theta_{2}} \theta_{2}+\gamma_{\theta_{2}} S_{\varphi} \psi_{2}\left(\bar{x}_{2}\right)$

$\dot{\theta}_{3}=-\sigma_{\theta_{3}} \gamma_{\theta_{3}} \theta_{3}+\gamma_{\theta_{3}} S_{i s d 1} \psi_{3}\left(\bar{x}_{3}\right)$

$\dot{\theta}_{4}=-\sigma_{\theta_{4}} \gamma_{\theta_{4}} \theta_{4}+\gamma_{\theta_{4}} S_{i s q 1} \psi_{4}\left(\bar{x}_{4}\right)$

$\dot{\theta}_{5}=-\sigma_{\theta_{5}} \gamma_{\theta_{5}} \theta_{5}+\gamma_{\theta_{5}} S_{i s o 1} \psi_{5}\left(\bar{x}_{5}\right)$

$\dot{\theta}_{6}=-\sigma_{\theta_{6}} \gamma_{\theta_{6}} \theta_{6}+\gamma_{\theta_{6}} S_{i s d 2} \psi_{6}\left(\bar{x}_{6}\right)$

$\dot{\theta}_{7}=-\sigma_{\theta_{7}} \gamma_{\theta_{7}} \theta_{7}+\gamma_{\theta_{7}} S_{\text {isq } 2} \psi_{7}\left(\bar{x}_{7}\right)$

$\dot{\theta}_{8}=-\sigma_{\theta_{8}} \gamma_{\theta_{8}} \theta_{8}+\gamma_{\theta_{8}} S_{i s o 2} \psi_{8}\left(\bar{x}_{8}\right)$

$\dot{k}_{12}=-\sigma_{k_{1}} \gamma_{k_{1}} k_{12}+\gamma_{k_{1}} S_{\Omega} \tanh \left(\frac{s_{\Omega}}{\varepsilon_{i s q}}\right)$

$\dot{k}_{22}=-\sigma_{k_{2}} \gamma_{k_{2}} k_{22}+\gamma_{k_{2}} S_{\varphi} \tanh \left(\frac{S_{\varphi}}{\varepsilon_{i s d}}\right)$

$\dot{k}_{32}=-\sigma_{k_{3}} \gamma_{k_{3}} k_{32}+\gamma_{k_{3}} S_{i s d 1} \tanh \left(\frac{S_{i s d 1}}{\varepsilon_{i s d 1}}\right)$

$\dot{k}_{42}=-\sigma_{k_{4}} \gamma_{k_{4}} k_{42}+\gamma_{k_{4}} S_{\text {isq1 }} \tanh \left(\frac{s_{i s q 1}}{\varepsilon_{i s q 1}}\right)$

$\dot{k}_{52}=-\sigma_{k_{5}} \gamma_{k_{5}} k_{52}+\gamma_{k_{5}} S_{\text {iso1 }} \tanh \left(\frac{S_{i s o 1}}{\varepsilon_{i s o 1}}\right)$

$\dot{k}_{62}=-\sigma_{k_{6}} \gamma_{k_{6}} k_{62}+\gamma_{k_{6}} S_{i s d 2} \tanh \left(\frac{S_{i s d 2}}{\varepsilon_{i s d 2}}\right)$

$\dot{k}_{72}=-\sigma_{k_{7}} \gamma_{k_{7}} k_{72}+\gamma_{k_{7}} S_{i s q 2} \tanh \left(\frac{s_{i s q 2}}{\varepsilon_{i s q 2}}\right)$

$\dot{k}_{82}=-\sigma_{k_{8}} \gamma_{k_{8}} k_{82}+\gamma_{k_{8}} S_{\text {iso2 }} \tanh \left(\frac{S_{i s o 2}}{\varepsilon_{\text {iso2 }}}\right)$

Where: $\sigma_{\theta_{i}}, \gamma_{\theta_{i}}, \gamma_{k_{i}}, \sigma_{k_{i}}>0$ (for $i=\overline{1,8}$ ); these parameters are design constants.

\section{Theorem 1}

The following properties are valid for DSIM modeled by (5)-(12) and controlled by the adaptive laws presented in (34)(49):

The signals delimitation is guaranteed in closed-loop.

The optimal choice of the setting parameters ensures the exponential convergence of the errors variables $\widetilde{\Omega}(t), \tilde{\varphi}_{r}(t)$, $\tilde{i}_{s d 1}(t), \tilde{i}_{s q 1}(t), \tilde{i}_{s d 2}(t), \tilde{i}_{s q 2}(t), \tilde{l}_{s o 1}(t)$ and $\tilde{i}_{s o 2}(t)$ to a ball with an insignificant radius.

The proof of Theorem 1 is based on Lyapunov's theory of stability. It is presented by a feedback structure with two consecutive steps.

Step 1: The purpose of this step is to lead the speed to its desired reference by an adequate speed controller. Using the formula of the filtered rotor speed error defined in (17): 
$S_{\Omega}=\tilde{\Omega}(t)+\lambda_{\Omega} \int_{0}^{t} \tilde{\Omega}(\tau) d \tau$

Using 13, the time derivative of $S_{\Omega}$ is:

$\dot{S}_{\Omega}=\dot{\tilde{\Omega}}(t)+\lambda_{\Omega} \tilde{\Omega}$

$\dot{S}_{\Omega}=\frac{p^{2}}{J} \frac{L_{m}}{L_{m}+L_{r}} \varphi_{r}\left(i_{s q 1}+i_{s q 2}\right)+f_{1}-\dot{\Omega}^{*}+\lambda_{\Omega} \tilde{\Omega}$

$\dot{S}_{\Omega}=h_{1}\left(\bar{x}_{1}\right)+\frac{p^{2}}{J} \frac{L_{m}}{L_{m}+L_{r}} \varphi_{r} i_{s q}^{*}$

where: $h_{1}\left(\bar{x}_{1}\right)=f_{1}-\dot{\Omega}^{*}+\lambda_{\Omega} \tilde{\Omega}$ and $i_{s q}^{*}$ is the reference value of $\left(i_{s q 1}+i_{s q 2}\right)$ that regulates the rotor speed and ensures the load disturbances rejection capability. The Lyapunov function associated with the rotor speed error is presented by:

$V_{1}=\frac{1}{2} S_{\Omega}^{2}$

The time derivative of (52) is:

$\dot{V}_{1}=S_{\Omega} h_{1}\left(\bar{x}_{1}\right)+\frac{p^{2}}{J} \frac{L_{m}}{L_{m}+L_{r}} \varphi_{r} i_{s q}^{*}$

The following adaptive fuzzy system is developed to approximate the uncertain continuous function $h_{1}\left(x_{1}\right)$ :

$\hat{h}_{1}\left(\bar{x}_{1}\right)=\theta_{1}^{T} \psi_{1}\left(\bar{x}_{1}\right)$

$h_{1}\left(\bar{x}_{1}\right)=\theta_{1}^{* T} \psi_{1}\left(\bar{x}_{1}\right)+\bar{\omega}_{1}\left(\bar{x}_{1}\right)$

$h_{1}\left(\bar{x}_{1}\right)=-\tilde{\theta}_{1}^{T} \psi_{1}\left(\bar{x}_{1}\right)+\theta_{1}^{T} \psi_{1}\left(\bar{x}_{1}\right)+\bar{\omega}_{1}\left(\bar{x}_{1}\right)$

where: $\tilde{\theta}_{1}=\theta_{1}-\theta_{1}^{*}$ is the parameter error vector. By replacing (55) in (53), we obtain:

$\dot{V}_{1}=-S_{\Omega} \tilde{\theta}_{1}^{T} \psi_{1}\left(\bar{x}_{1}\right)+S_{\Omega} \theta_{1}^{T} \psi_{1}\left(\bar{x}_{1}\right)+S_{\Omega} \bar{\omega}_{1}\left(\bar{x}_{1}\right)+$

$\frac{p^{2}}{J} \frac{L_{m}}{L_{m}+L_{r}} \varphi_{r} i_{s q}^{*}$

where: $\overline{\bar{\omega}}_{1}$ is an unknown constant such as $\left|\bar{\omega}_{1}\left(\bar{x}_{1}\right)\right| \leq \overline{\bar{\omega}}_{1}$.

By choosing the expression of $i_{s q}^{*}$ presented in (26), we can make the following inequality:

$\dot{V}_{1} \leq-S_{\Omega} \tilde{\theta}_{1}^{T} \psi_{1}\left(\bar{x}_{1}\right)+k_{12}^{*}\left|S_{\Omega}\right|-k_{12} S_{\Omega} \tanh \left(\frac{s_{\Omega}}{\varepsilon_{i s q}}\right)-k_{11} S_{\Omega}^{2}$

where: $k_{12}^{*}=\overline{\bar{\omega}}_{1}$.

Lemma 1 the set $\left\{\varepsilon_{i}>0, x \in \Re\right\}$ check the following inequality [11]:

$\left\{\begin{array}{l}0 \leq|x|-x \tanh \left(\frac{x}{\varepsilon_{i}}\right) \leq \varepsilon_{i}=\rho \varepsilon_{i} \\ p=e^{-(1+p)} \simeq 0.2785\end{array}\right.$

By exploiting (58), (57) becomes:

$\dot{V}_{1} \leq-S_{\Omega} \tilde{\theta}_{1}^{T} \psi_{1}\left(\bar{x}_{1}\right)+k_{12}^{*} \bar{\varepsilon}_{i s q}-\tilde{k}_{12} S_{\Omega} \tanh \left(\frac{s_{\Omega}}{\varepsilon_{i s q}}\right)-k_{11} S_{\Omega}^{2}$

where $\bar{k}_{12}=k_{12}-k_{12}^{*}$ and $\bar{\varepsilon}_{i s q}=0.2785 \varepsilon_{i s q}$.

The Lyapunov function linked to the adaptive laws that estimate the unknown parameters $\theta_{1}^{*}$ and $k_{12}^{*}$ is defined by:

$V_{2}=V_{1}+\frac{1}{2 \gamma_{\theta_{1}}} \tilde{\theta}_{1}^{T} \tilde{\theta}_{1}+\frac{1}{2 \gamma_{k_{1}}} \tilde{k}_{12}^{2}$
The dynamics of Lyapunov function verify the following inequality:

$\dot{V}_{2} \leq-S_{\Omega} \tilde{\theta}_{1}^{T} \psi_{1}\left(\bar{x}_{1}\right)+k_{12}^{*} \bar{\varepsilon}_{i s q}-\tilde{k}_{12} S_{\Omega} \tanh \left(\frac{s_{\Omega}}{\varepsilon_{i s q}}\right)-$

$k_{11} S_{\Omega}^{2}+\frac{1}{2 \gamma_{\theta_{1}}} \tilde{\theta}_{1}^{T} \dot{\theta}_{1}+\frac{1}{2 \gamma_{k_{1}}} \tilde{k}_{12} \dot{k}_{12}$

By substituting the values of $\dot{\theta}_{1}$ and $\dot{k}_{12}$ chosen in (34)-(41) and (42)-(49), respectively, $\dot{V}_{2}$ will be bounded by the following expression:

$\dot{V}_{2} \leq k_{12}^{*} \bar{\varepsilon}_{i s q}-k_{11} S_{\Omega}^{2}-\sigma_{\theta_{1}} \tilde{\theta}_{1}^{T} \theta_{1}-\sigma_{k_{1}} \tilde{k}_{12} k_{12}$

\section{Property:}

$\left\{\begin{array}{l}-\widetilde{\Theta}^{T} \Theta \leq-\frac{1}{2}\|\widetilde{\Theta}\|^{2}+\frac{1}{2}\left\|\Theta^{*}\right\|^{2} \\ \widetilde{\Theta}=\Theta-\Theta^{*} \in \mathfrak{R}^{m}\end{array}\right.$

where: $\mathrm{m}$ is a positive integer number. By using (63), (62) takes the following form:

$\dot{V}_{2} \leq-k_{11} S_{\Omega}^{2}-\frac{\sigma_{\theta_{1}}}{2}\left\|\tilde{\theta}_{1}\right\|^{2}-\frac{\sigma_{k_{1}}}{2} \tilde{k}_{12}^{2}+\bar{\varepsilon}_{1}$

With: $\bar{\varepsilon}_{1}=k_{12}^{*} \bar{\varepsilon}_{i s q}+\frac{\sigma_{\theta_{1}}}{2}\left\|\theta_{1}^{*}\right\|^{2}+\frac{\sigma_{k_{1}}}{2} k_{12}^{* 2}$

The stabilization of the filtered errors $S_{\varphi}, S_{i s d 1}, S_{i s q 1}, S_{i s o 1}, S_{i s d 2}, S_{i s q 2}$, and $S_{s o 2}$ will be achieved in the next step.

Step 2: The aim of this step is to design the following control laws: $i_{s d}^{*}, v_{s d 1}, v_{s q 1}, v_{s o 1}, v_{s d 2}, v_{s q 2}$ and $v_{s o 2}$.

The Lyapunov function adapted to this step is given by:

$V_{3}=V_{2}+\frac{1}{2} S_{\varphi}^{2}+\frac{1}{2} S_{i s d 1}^{2}+\frac{1}{2} S_{i s q 1}^{2}+\frac{1}{2} S_{i s o 1}^{2}+\frac{1}{2} S_{i s d 2}^{2}+$ $\frac{1}{2} S_{i s q 2}^{2}+\frac{1}{2} S_{i s o 2}^{2}$

The dynamics of the Lyapunov function verify the following inequality:

$\dot{V}_{3} \leq-k_{11} S_{\Omega}^{2}-\frac{\sigma_{\theta_{1}}}{2}\left\|\tilde{\theta}_{1}\right\|^{2}-\frac{\sigma_{k_{1}}}{2} \tilde{k}_{12}^{2}+\bar{\varepsilon}_{1}+S_{\varphi} \dot{S}_{\varphi}+$

$S_{i s d 1} \dot{S}_{i s d 1}+S_{i s q 1} \dot{S}_{i s q 1}+S_{i s o 1} \dot{S}_{i s o 1}+S_{i s d 2} \dot{S}_{i s d 2}+$

$S_{i s q 2} \dot{S}_{i s q 2}+S_{i s o 2} \dot{S}_{i s o 2}$

The derivatives of the filtered errors are obtained using (5)(12) and (17)-(24):

$\dot{S}_{\varphi}=\frac{L_{m} R_{r}}{L_{m}+L_{r}} i_{s q}^{*}+f_{2}+\lambda_{\varphi} \widetilde{\varphi}_{r}-\dot{\varphi}_{r}^{*}$

$\dot{S}_{i s d 1}=\frac{1}{L_{s 1}} v_{s d 1}+f_{3}+\lambda_{i s d 1} \tilde{l}_{s d 1}-i_{s d 1}^{*}$

$\dot{S}_{i s q 1}=\frac{1}{L_{s 1}} v_{s q 1}+f_{4}+\lambda_{i s q 1} \tilde{I}_{s q 1}-i_{s q 1}^{*}$

$\dot{S}_{i s o 1}=\frac{1}{L_{s 1}} v_{s o 1}+f_{5}+\lambda_{i s o 1} \tilde{l}_{s o 1}-i_{s o 1}^{*}$

$\dot{S}_{i s d 2}=\frac{1}{L_{s 2}} v_{s d 2}+f_{6}+\lambda_{i s d 2} \tilde{l}_{s d 2}-i_{s d 2}^{*}$

$\dot{S}_{i s q 2}=\frac{1}{L_{s 2}} v_{s q 2}+f_{7}+\lambda_{i s q 2} \tilde{l}_{s q 2}-i_{s q 2}^{*}$ 
$\dot{S}_{i s o 2}=\frac{1}{L_{s 2}} v_{s o 2}+f_{8}+\lambda_{i s o 2} \tilde{l}_{s o 2}-i_{s o 2}^{*}$

By replacing (67-73) in (66), we obtain:

$\dot{V}_{3} \leq-k_{11} S_{\Omega}^{2}-\frac{\sigma_{\theta_{1}}}{2}\left\|\tilde{\theta}_{1}\right\|^{2}-\frac{\sigma_{k_{1}}}{2} \tilde{k}_{12}^{2}+\bar{\varepsilon}_{1}+S_{\varphi}\left(h_{2}\left(\bar{x}_{2}\right)+\right.$ $\left.\frac{L_{m} R_{r}}{L_{m}+L_{r}} i_{s q}^{*}\right)+S_{i s d 1}\left(h_{3}\left(\bar{x}_{3}\right)+\frac{1}{L_{s 1}} v_{s d 1}\right)+S_{i s q 1}\left(h_{4}\left(\bar{x}_{4}\right)+\right.$ $\left.\frac{1}{L_{s 1}} v_{s q 1}\right)+S_{i s o 1}\left(h_{5}\left(\bar{x}_{5}\right)+\frac{1}{L_{s 1}} v_{s o 1}\right)+S_{i s d 2}\left(h_{6}\left(\bar{x}_{6}\right)+\right.$ $\left.\frac{1}{L_{s 2}} v_{s d 2}\right)+S_{i s q 2}\left(h_{7}\left(\bar{x}_{7}\right)+\frac{1}{L_{s 2}} v_{s q 2}\right)+S_{i s o 2}\left(h_{8}\left(\bar{x}_{8}\right)+\right.$ $\left.\frac{1}{L_{S 2}} v_{S o 2}\right)$

With:

$h_{2}\left(\bar{x}_{2}\right)=f_{2}+\lambda_{\varphi} \tilde{\varphi}_{r}-\dot{\varphi}_{r}^{*}$

$h_{3}\left(\bar{x}_{3}\right)=f_{3}+\lambda_{i s d 1} \tilde{l}_{s d 1}-\tilde{l}_{s d 1}^{*}$

$h_{4}\left(\bar{x}_{4}\right)=f_{4}+\lambda_{i s q 1} \tilde{r}_{s q 1}-\tilde{i}_{s q 1}^{*}$

$h_{5}\left(\bar{x}_{5}\right)=f_{5}+\lambda_{i s o 1} \tilde{l}_{s o 1}-\tilde{i}_{s o 1}^{*}$

$h_{6}\left(\bar{x}_{6}\right)=f_{6}+\lambda_{i s d 2} \tilde{l}_{s d 2}-\tilde{l}_{s d 2}^{*}$

$h_{7}\left(\bar{x}_{7}\right)=f_{7}+\lambda_{i s q 2} \tilde{l}_{s q 2}-\tilde{l}_{s q 2}^{*}$

$h_{8}\left(\bar{x}_{8}\right)=f_{8}+\lambda_{i s o 2} \tilde{l}_{s o 2}-\tilde{l}_{s o 2}^{*}$

$h_{i}\left(\bar{x}_{i}\right), i=\overline{2,8}$ are continuous uncertainties functions, their approximation is performed by the following adaptive fuzzy system:

$\widehat{h}_{i}\left(\bar{x}_{i}\right)=\theta_{i}^{T} \psi_{i}\left(\bar{x}_{i}\right)$

$h_{i}\left(\bar{x}_{i}\right)=\theta_{i}^{* T} \psi_{i}\left(\bar{x}_{i}\right)+\bar{\omega}_{i}\left(\bar{x}_{i}\right)$

$h_{i}\left(\bar{x}_{i}\right)=-\tilde{\theta}_{i}^{T} \psi_{i}\left(\bar{x}_{i}\right)+\theta_{i}^{T} \psi_{i}\left(\bar{x}_{i}\right)+\bar{\omega}_{i}\left(\bar{x}_{i}\right)$ for $i=\overline{1,8}$

where: $\tilde{\theta}_{i}=\theta_{i}-\theta_{i}^{*}$ expresses the error vector, $\bar{x}_{i}$ is predefined, $\bar{\omega}_{i}\left(\bar{x}_{i}\right)$ is the fuzzy approximation error with $\bar{\omega}_{i}\left(\bar{x}_{i}\right) \leq \overline{\bar{\omega}}_{i}, \forall \bar{x}_{i} \in D_{x_{i}}, \overline{\bar{\omega}}_{i}$ is an unknown constant.

$\left\{\theta_{i}^{*}, k_{i 2}^{*}\right\}, i=\overline{2,8}$ are unknown parameters, their estimation requires an adaptive law defined by the following Lyapunov function:

$V_{4}=V_{3}+\frac{1}{2 \gamma_{\theta_{2}}} \tilde{\theta}_{2}^{T} \tilde{\theta}_{2}+\frac{1}{2 \gamma_{k_{2}}} \tilde{k}_{22}^{2}+\frac{1}{2 \gamma_{\theta_{3}}} \tilde{\theta}_{3}^{T} \tilde{\theta}_{3}+\frac{1}{2 \gamma_{k_{3}}} \tilde{k}_{32}^{2}+$ $\frac{1}{2 \gamma_{\theta_{4}}} \tilde{\theta}_{4}^{T} \tilde{\theta}_{4}+\frac{1}{2 \gamma_{k_{4}}} \tilde{k}_{42}^{2}+\frac{1}{2 \gamma_{\theta_{5}}} \tilde{\theta}_{5}^{T} \tilde{\theta}_{5}+\frac{1}{2 \gamma_{k_{5}}} \tilde{k}_{52}^{2}+\frac{1}{2 \gamma_{\theta_{6}}} \tilde{\theta}_{6}^{T} \tilde{\theta}_{6}+$

$\frac{1}{2 \gamma_{k_{6}}} \tilde{k}_{62}^{2}+\frac{1}{2 \gamma_{\theta_{7}}} \tilde{\theta}_{7}^{T} \tilde{\theta}_{7}+\frac{1}{2 \gamma_{k_{7}}} \tilde{k}_{72}^{2}+\frac{1}{2 \gamma_{\theta_{8}}} \tilde{\theta}_{8}^{T} \tilde{\theta}_{8}+\frac{1}{2 \gamma_{k_{8}}} \tilde{k}_{82}^{2}$

The derivation of (78) gives (see Appendix B):

$\dot{V}_{4} \leq-\eta V_{4}+\mu$

With:

$\mu=\bar{\varepsilon}_{1}+\bar{\varepsilon}_{2}+\bar{\varepsilon}_{3}+\bar{\varepsilon}_{4}+\bar{\varepsilon}_{5}+\bar{\varepsilon}_{6}+\bar{\varepsilon}_{7}+\bar{\varepsilon}_{8}$

$\eta_{k}=\min \left\{\sigma_{k_{1}} \gamma_{k_{1}}, \sigma_{k_{2}} \gamma_{k_{2}}, \sigma_{k_{3}} \gamma_{k_{3}}, \sigma_{k_{4}} \gamma_{k_{4}}, \sigma_{k_{5}} \gamma_{k_{5}}, \sigma_{k_{6}}\right.$

$\left.\gamma_{k_{6}}, \sigma_{k_{7}} \gamma_{k_{7}}, \sigma_{k_{8}} \gamma_{k_{8}}\right\}$

And

$\eta=$

$\min \left\{2 k_{11}, 2 k_{21}, 2 k_{31}, 2 k_{41}, 2 k_{51}, 2 k_{61}, 2 k_{71}, 2 k_{81}, \sigma_{\theta_{1}}\right.$

$\left.\gamma_{\theta_{1}}, \sigma_{\theta_{2}} \gamma_{\theta_{2}}, \sigma_{\theta_{3}} \gamma_{\theta_{3}}, \sigma_{\theta_{4}} \gamma_{\theta_{4}}, \sigma_{\theta_{5}} \gamma_{\theta_{5}}, \sigma_{\theta_{6}} \gamma_{\theta_{6}}, \sigma_{\theta_{7}} \gamma_{\theta_{7}}, \sigma_{\theta_{8}} \gamma_{\theta_{8}}, \eta_{k}\right\}$

If we multiply (79) by the exponential term $e^{\eta t}$, we obtain [11]: $\frac{d}{d t}\left(V_{4} e^{\eta t}\right) \leq \mu e^{\eta t}$

The integration of (80) from 0 to $t$ gives us:

$0 \leq V_{4} \leq \frac{\mu}{\eta}+\left(V_{4}(0)-\frac{\mu}{\eta}\right) e^{-\eta t}$

where: $\mu \mathrm{s}$ a randomly selected parameter and $\eta$ is chosen according to the design parameters. According to [11]: the bounded interval of $\dot{V}_{4}$ presented by (81) reflects the exponential convergence to an adaptable residual set for tracking errors, filtered tracking errors and parameter estimation errors, adding to that the delimitation of all closedloop signals. The global block diagram of the proposed FTC is shown in Figure 2.

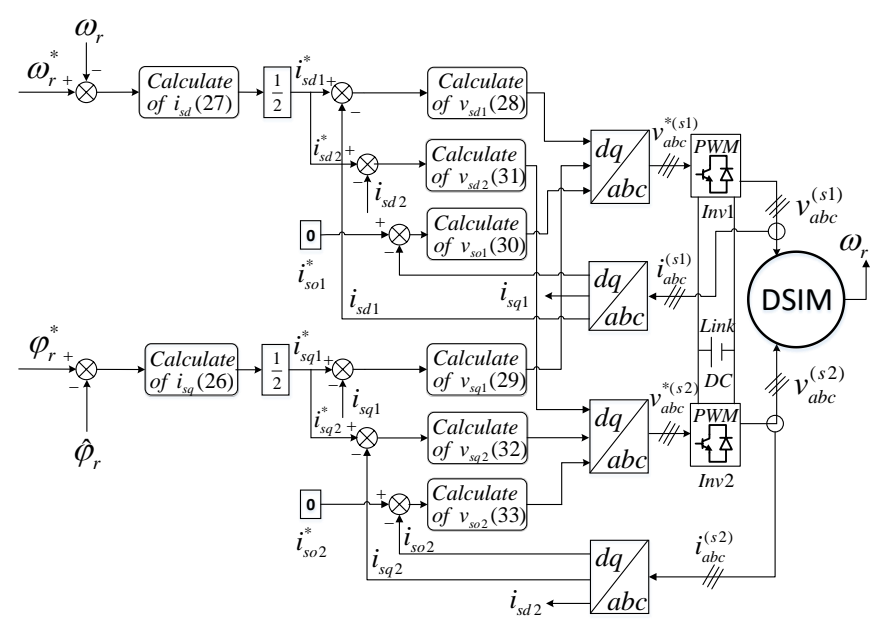

Figure 2. lock diagram of the proposed FTC

\section{SIMULATION RESULTS AND COMPARISONS}

\subsection{Fault diagnostic in open loop}

The machine parameters used to simulate the DSIM faulty model presented in section 2 are given in Appendix A. The DSIM studied in this paper is powered by two three-phase voltage source inverters (VSIs) using pulse width modulation (PWM) control strategies. In steady-state conditions, the DSIM operates with a fundamental frequency equal to $50 \mathrm{~Hz}$ at $100 \%$ load. Figure 3 below shows the evolution in time of the speed, the electromagnetic torque, the stator current and the flux for DSIM operating in open loop with a healthy and defective squirrel cage rotor.

The vectors $\bar{x}_{1}=\left[\Omega^{*}, \Omega\right]^{T}, \quad \bar{x}_{2}=\left[\varphi_{r}^{*}, \varphi_{r}\right]^{T}, \quad \bar{x}_{3}=$ $\left[i_{s d 1}, i_{s q 1}\right]^{T}, \bar{x}_{4}=\left[i_{s q 1}, i_{s d 1}\right]^{T}, \bar{x}_{5}=\left[i_{s o 1}^{*}, i_{s o 1}\right]^{T}, \bar{x}_{6}=$ $\left[i_{s d 2}, i_{s q 2}\right]^{T}, \quad \bar{x}_{7}=\left[i_{s q 2}, i_{s d 2}\right]^{T} \quad$ and $\quad \bar{x}_{8}=\left[i_{s o 2}^{*}, i_{s o 2}\right]^{T}$ present the inputs of the type-2 fuzzy system $\theta_{i}^{T} \psi_{i}\left(\bar{x}_{i}\right)$ for $i=$ $\overline{1,8}$.

For the variables $\left(\Omega, \Omega^{*}, \varphi_{r}, \varphi_{r}^{*}\right)$ and $\left(i_{s d 1}, i_{s q 1}, i_{s d 2}, i_{s q 2}, i_{s o 1}^{*}, i_{s o 1}, i_{s o 2}^{*}, i_{s o 2}\right)$ we define five type-2 Gaussian membership functions uniformly distributed over intervals $[-30,30]$ and $[-3,3]$ respectively. 


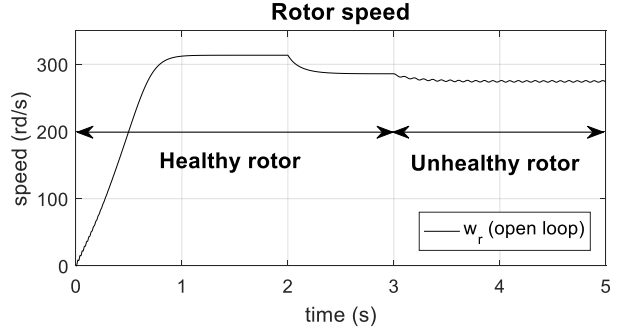

(a)

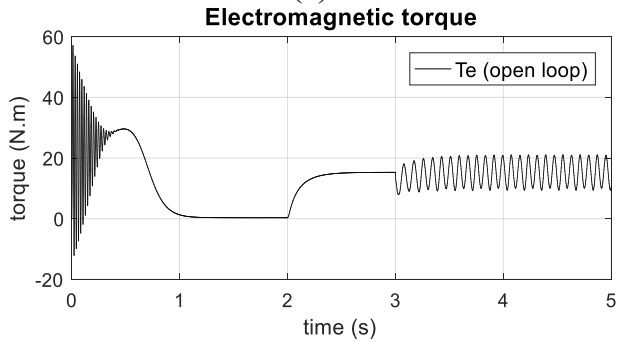

(b)

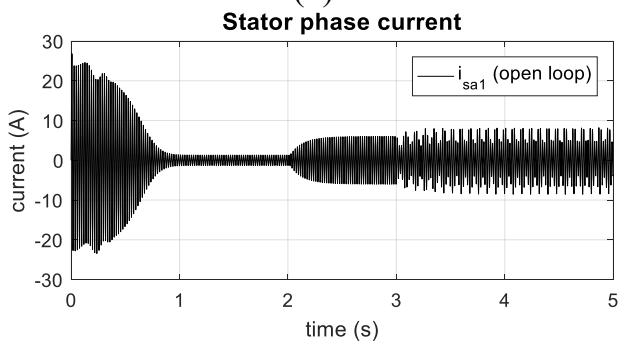

(c)

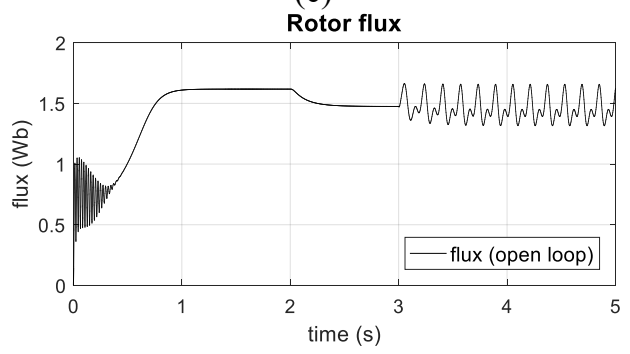

(d)

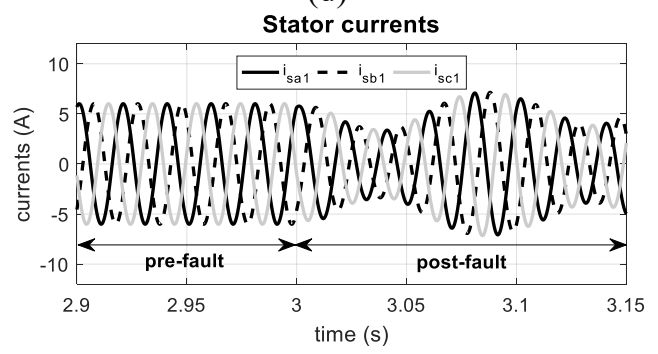

(e)

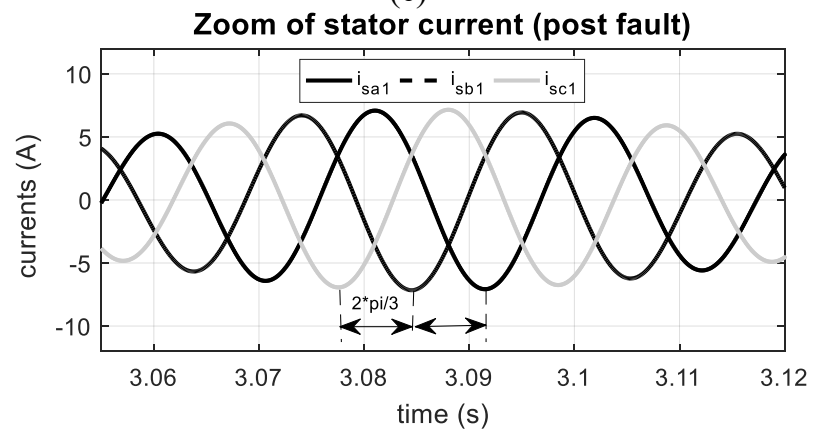

(f)

Figure 3. Evolution of the parameter curve, at startup, in load and when breaking bars
The startup is done empty under a nominal voltage with a balanced sinusoidal power supply. The time response is approximately equal to $1 \mathrm{~s}$. An external load torque of nominal value equal to $15 \mathrm{~N} . \mathrm{m}$ is applied at $\mathrm{t}=2 \mathrm{sec}$, the speed then drops to its nominal value of $286 \mathrm{rad} / \mathrm{s}$. The electromagnetic torque compensates the load torque and the friction losses. The current increases and reaches its nominal value at time $t=1 \mathrm{~s}$. A broken bars fault is caused at $t=3 \mathrm{sec}$ by increasing the resistance of a rotor phase $(e=6 \Omega)$.The fault appears instantaneously and the rotor circuit becomes asymmetrical because the rotor creates in addition to the direct field $\left(+s \omega_{s}\right)$, an inverse rotor field $\left(-s \omega_{s}\right)$, the interaction of these fields with that resulting from the stator winding produces an electromagnetic torque of pulsation $\left[V_{r}^{a b c}\right]=\left[R_{r}^{B R B}\right]\left[I_{r}^{a b c}\right]+$ $\frac{d}{d t}\left[\Phi_{r}^{a b c}\right]$, as a result, the value of the electromagnetic torque will be equal to the sum of a constant component and a sinusoidal inverse component, resulting in the reduction of the average value of the electromagnetic torque and the appearance of periodic oscillations in the speed signal and torque, as shown in Figure 3.a and Figure 3.b respectively. Figure 3.c presents the stator current in which a weak modulation of the magnitude can be observed, the flux signal is also affected by oscillations related to the BRB fault (see Figure 3.d). Figure 3.e and Figure 3.f show that the stator phase currents are always shifted by $\frac{2 \pi}{3}$ but ripples in their amplitudes appear with the occurrence of BRB. All these diagnoses prove the appearance of the BRB fault [17]. In the unbalanced machine winding, harmonics causes sidebands at specific frequencies around the supply frequency. In this study, the fast Fourier transform (FFT) is used in order to distinguish these frequency components from the stator current spectrum.

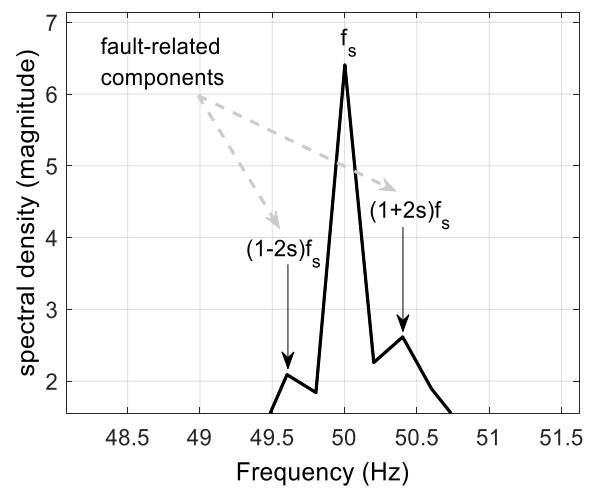

Figure 4. Stator current spectral density

Figure 4 presents the spectral analysis of the stator current in steady state using the FFT method when the DSIM operates under nominal load for a slip $\mathrm{s}=0.4 \%$. When the DSIM operates with $\mathrm{BRB}$ fault sidebands at $(1 \pm 2 s) f_{s}$ appear around the supply frequency. This result is a reliable signature of the BRB fault. Moreover, the amplitude of this harmonics is an indication of the degree of severity of the fault.

\subsection{Healthy and defective states of DSIM in closed loop}

The efficiency and robustness of the proposed control compared to SMC proposed in Listwan and Fnaiech [9-10] with different modes of operation, especially in post-fault operation are shown through simulation results using MATLAB/SIMULINK. The reference speed is set at $200 \mathrm{rd} / \mathrm{s}$, the BRB fault is introduced at $\mathrm{t}=2 \mathrm{sec}$. The simulations 
presented in Figure 5 show the DSIM responses in healthy and defective mode with SMC and proposed FTC. The results showed the superior performance of the proposed FTC based on IT2FLS.

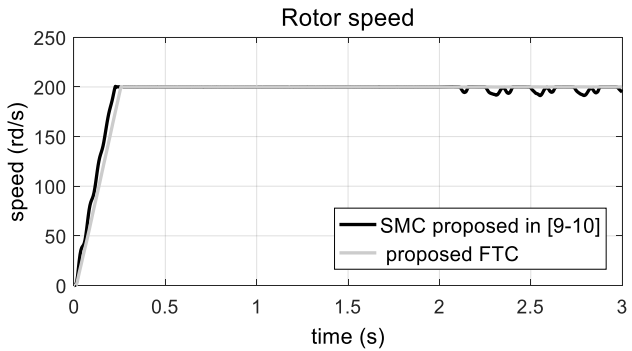

(a)

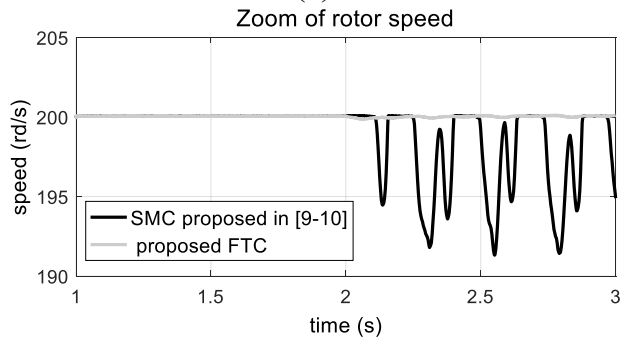

(b)

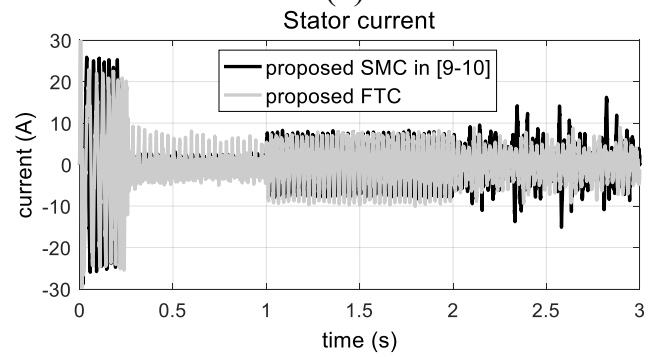

(c)

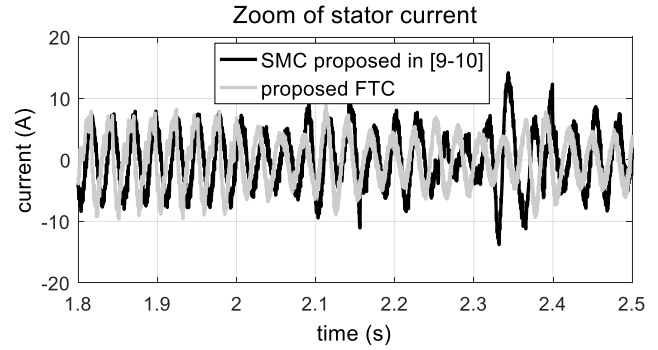

(d)

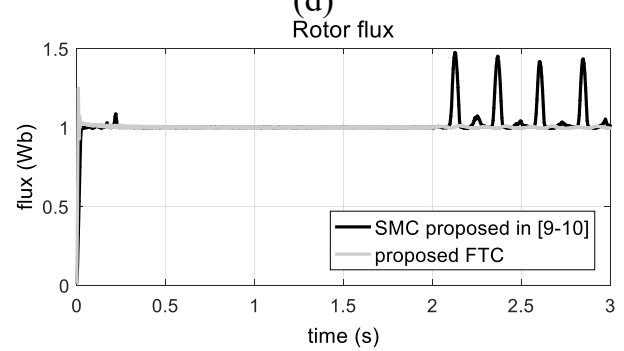

(e)

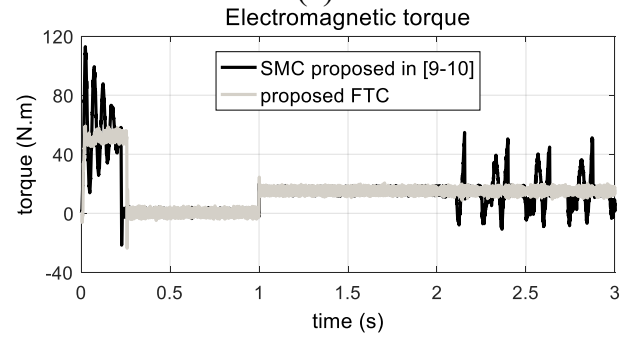

(f)

Figure 5. Pre-fault $(\mathrm{t}<2 \mathrm{~s})$ and post-fault $(\mathrm{t}>2 \mathrm{~s})$ performance of SMC proposed in [9-10] and proposed FTC for DSIM
The DSIM is starting with a balanced squirrel cage rotor from zero to the nominal speed, at $\mathrm{t}=1 \mathrm{~s}$, DSIM is loaded by $T_{L}=T_{L N}=15 \mathrm{~N} . \mathrm{m}$, a simulation of the BRB fault is caused at $\mathrm{t}=2 \mathrm{~s}$. During the un-faulty mode, the speed follows its reference value with a negligible overshoot and without oscillations, but it is clearly shows that SMC has a faster dynamic response than the proposed FTC by imposing a short transient regime, the load torque is very well compensated by the electromagnetic torque (before $\mathrm{t}=2 \mathrm{~s}$ ). It is clear that after the fault occurrence, an abnormal behavior of the DSIM is observed with SMC accompanied by closed-loop performance degradation; velocity oscillations are visible through the zoom presented in Figure 5.a and Figure 5.b. The stator phase current is not sinusoidal, the distortion of the signal is caused by the fault effect, the oscillations on this physical quantity are visible in Figure 5.c and Figure 5.d, their amplitude can reach up to \pm 14 A greater than the nominal value of the current. The flux trajectory is presented in Figure 5.e; SMC provides ripples after the appearance of the BRB fault. High ripples in the electromagnetic torque can be view in Figure 5.f, where the maximum positive ripple reaches $+55 \mathrm{~N}$.m and the maximum negative ripple reaches -11 N.m. Regarding the proposed FTC, oscillations in rotor speed are considerably reduced as indicated by the Figure 5.a and Figure 5.b, the proposed FTC guarantees a better speed response with precise reference tracking and also provides better stability with the smallest average static error. The tracking performance of the stator current has a small change, the current signal is not sinusoidal but does not exceed its nominal value, this deformation represented in Figure 5.d expresses the compensation of the BRB fault effect by the stators phases. Figure 5.e proves that the proposed FTC is able to correctly lead the flux with a fast dynamic to its desired reference $(1 \mathrm{~Wb})$ even under rotor fault. No ripple in the electromagnetic torque signal during faulty operation as shown in Figure 5.f. Finally, it can be seen from the simulations results that the BRB fault does not affect the performance of the proposed FTC even in the presence of load torque while SMC proposed in Listwan and Fnaiech is unable to properly handle the machine with an unbalanced rotor $[9,10]$.

\section{CONCLUSION}

DSIM's high-impact in the industry requires fault-tolerant performance. This can be achieved by a fuzzy adaptive control using the IT2FLS; the proposed FTC maintains maximum performance of the double star induction machine even under broken bars fault. The effectiveness of the proposed FTC is validated using MATLAB/SIMULINK. The obtained results show that the proposed fault-tolerant approach is able to handle the post-fault operation and provides satisfactory performance in terms of speed and torque responses even under such abnormal conditions. In addition, the comparative study performed with other work recently developed on a multiphase machine has shown improved fault tolerant performance. This adaptive fault-tolerant control could be a realistic solution and a powerful alternative to the existing FTC methods.

\section{REFERENCES}

[1] Layadi N, Zeghlache S, Benslimane T, Berrabah F. 
(2017). Comparative analysis between the rotor flux oriented control and backstepping control of a double star induction machine (DSIM) under open-phase fault. AMSE Journals, Series Advances C 72(4): 292-311.

[2] Rouaibia R, Arbaoui F, Bahi T. (2017). Sliding fault eccentricity diagnosis in variable speed induction motor drive using DWT. AMSE Journals, Series Advances C 72(3): 181-202.

[3] Lizarraga-Morales RA, Rodriguez-Donate C, CabalYepez E, Lopez-Ramirez M, Ledesma-Carrillo LM, Ferrucho-Alvarez ER. (2017). Novel FPGA-based methodology for early broken rotor bar detection and classification through homogeneity estimation. IEEE Transactions on Instrumentation and Measurement 66(7): 1760-1769. https://doi.org/10.1109/TIM.2017.2664520

[4] Elbouchikhi E, Choqueuse V, Auger F, Benbouzid MEH. (2017). Motor current signal analysis based on a matched subspace detector. IEEE Transactions on Instrumentation and Measurement 66(12): 3260-3270. https://doi.org/10.1109/TIM.2017.2749858

[5] Hou Z, Huang J, Liu H, Wang T, Zhao L. (2016). Quantitative broken rotor bar fault detection for closedloop controlled induction motors. IET Electric Power Applications 10(5): 403-410. https://doi.org/10.1049/ietepa.2015.0440

[6] Belhamdi S, Goléa A. (2017). Fuzzy sliding mode speed controller design of induction motor drives with broken bars. AMSE Journals, Series Advances C 72(4): 281-291.

[7] Sousa KM, da Costa IBV, Maciel ES, Rocha JE, Martelli C, da Silva JCC. (2017). Broken bar fault detection in induction motor by using optical fiber strain sensors. IEEE Sensors Journal 17(12): 3669-3676. https://doi.org/10.1109/JSEN.2017.2695961

[8] Belhamdi S, Goléa A. (2017). Direct field-oriented control using fuzzy logic Type-2 for induction motor with broken rotor bars. AMSE Journals, Series Advances C 72(4): 203-212.

[9] Listwan J, Pieńkowski K. (2016). Sliding-mode direct field-oriented control of six-phase induction motor. Technical Transactions (2-M): 95-108. https://doi.org/10.5277/PED160106

[10] Betin FF, Capolino GA, Fnaiech F. (2010). Fuzzy logic and sliding-mode controls applied to six-phase induction machine with open phases. IEEE Transactions on Industrial Electronics 57(1): 354-364. https://doi.org/10.1109/TIE.2009.2034285

[11] Bounar N, Boulkroune A, Boudjema F, Farza M. (2015). Adaptive fuzzy vector control for a doubly-fed induction motor. Neurocomputing 151(2): 756-769. https://doi.org/10.1016/j.neucom.2014.10.026

[12] Mekki H, Benzineb O, Boukhetala D, TadjineM, Benbouzid M. (2015). Sliding mode based fault detection, reconstruction and fault tolerant control scheme for motor systems. ISA Transactions (57): 340351. https://doi.org/10.1016/j.isatra.2015.02.004

[13] Masumpoor S, Khanesar MA. (2015). Adaptive slidingmode type-2 neuro-fuzzy control of an induction motor. Expert Systems with Applications 42(19): 6635-6647. https://doi.org/10.1016/j.eswa.2015.04.046

[14] González-Prieto I, Duran MJ, Barrero FJ. (2017). Faulttolerant control of six-phase induction motor drives with variable current injection. IEEE Transactions on Power Electronics 32(10): 7894-7903. https://doi.org/10.1109/TPEL.2016.2639070
[15] Mahmoud EA, Abdel-Khalik AS, Soliman HF. (2016). An improved fault tolerant for a five-phase induction machine under open gate transistor faults. Alexandria Engineering Journal 55(3): 2609-2620. https://doi.org/10.1016/j.aej.2016.04.040

[16] Bednarz S. (2017). Rotor fault compensation and detection in a sensorless induction motor drive. Power Electronics and Drives 2(1): 71-80.

[17] Trujillo-Guajardo LA, Rodriguez-Maldonado J, Moonem MA, Platas-Garza MA. (2018). A multi resolution Taylor-Kalman approach for broken rotor bar detection in cage induction motors. IEEE Transactions on Instrumentation and Measurement 67(6): 1317-1328. https://doi.org/10.1109/TIM.2018.2795895

\section{APPENDIX A}

Machine parameters

$\mathrm{R}_{\mathrm{s} 1}=\mathrm{R}_{\mathrm{s} 2}=3.72 \Omega$ Stator 1 , Stator2 resistance respectively.

$\mathrm{L}_{\mathrm{s} 1}=\mathrm{L}_{\mathrm{s} 2}=0.022 \mathrm{H}$ Stator1, Stator2 inductance respectively.

$\mathrm{R}_{\mathrm{r}}=2.12 \Omega$ Rotor resistance.

$\mathrm{L}_{\mathrm{r}}=0.006 \mathrm{H}$ Rotor inductance.

$\mathrm{L}_{\mathrm{m}}=0.3672 \mathrm{H}$ Mutual inductance.

$\mathrm{J}=0.0625 \mathrm{Kgm}^{2}$ Moment of inertia

$\mathrm{K}_{\mathrm{f}}=0.001 \mathrm{Nm} .(\mathrm{rd} / \mathrm{s})^{-1}$ Viscous friction coefficient.

$\mathrm{p}=1$ Number of pole pairs

\section{APPENDIX B}

If we select the adaptive fuzzy controller components proposed in (26)-(33) and the continuous uncertainties functions $h_{i}\left(\bar{x}_{i}\right)$ developed in (77), $\dot{V}_{3}$ will be bounded by the following term:

$\dot{V}_{3} \leq-k_{11} S_{\Omega}^{2}-\frac{\sigma_{\theta_{1}}}{2}\left\|\tilde{\theta}_{1}\right\|^{2}-\frac{\sigma_{k_{1}}}{2} \tilde{k}_{12}^{2}+\bar{\varepsilon}_{1}-S_{\varphi} \tilde{\theta}_{2}^{T} \psi_{2}\left(\bar{x}_{2}\right)+$ $k_{22}^{*}\left|S_{\varphi}\right|-k_{22} S_{\varphi} \tanh \left(\frac{S_{\varphi}}{\varepsilon_{i s d}}\right)-k_{21} S_{\varphi}^{2}-S_{i s d 1} \tilde{\theta}_{3}^{T} \psi_{3}\left(\bar{x}_{3}\right)+$ $k_{32}^{*}\left|S_{i s d 1}\right|-k_{32} S_{i s d 1} \tanh \left(\frac{S_{i s d 1}}{\varepsilon_{i s d 1}}\right)-k_{31} S_{i s d 1}^{2}-$

$S_{i s q 1} \tilde{\theta}_{4}^{T} \psi_{4}\left(\bar{x}_{4}\right)+k_{42}^{*}\left|S_{i s q 1}\right|-k_{42} S_{i s q 1} \tanh \left(\frac{S_{i s q 1}}{\varepsilon_{i s q 1}}\right)-$

$k_{41} S_{i s q 1}^{2}-S_{i s o 1} \tilde{\theta}_{5}^{T} \psi_{5}\left(\bar{x}_{5}\right)+k_{52}^{*}\left|S_{i s o 1}\right|-$

$k_{52} S_{i s o 1} \tanh \left(\frac{S_{i s o 1}}{\varepsilon_{i s o 1}}\right)-k_{51} S_{i o s 1}^{2}-S_{i s d 2} \tilde{\theta}_{6}^{T} \psi_{6}\left(\bar{x}_{6}\right)+$

$k_{62}^{*}\left|S_{i s d 2}\right|-k_{62} S_{i s d 2} \tanh \left(\frac{S_{i s d 2}}{\varepsilon_{i s d 2}}\right)-k_{61} S_{i s d 2}^{2}-$

$S_{i s q 2} \tilde{\theta}_{7}^{T} \psi_{7}\left(\bar{x}_{7}\right)+k_{72}^{*}\left|S_{i s q 2}\right|-k_{72} S_{i s q 2} \tanh \left(\frac{s_{i s q 2}}{\varepsilon_{i s q 2}}\right)-$

$k_{71} S_{i s q 2}^{2}-S_{i s o 1} \tilde{\theta}_{8}^{T} \psi_{8}\left(\bar{x}_{8}\right)+k_{82}^{*}\left|S_{i s o 2}\right|-$

$k_{82} S_{i s o 2} \tanh \left(\frac{S_{i s o 2}}{\varepsilon_{i s o 2}}\right)-k_{81} S_{i s o 2}^{2}$

where: $k_{22}^{*}=\overline{\bar{\omega}}_{2}, k_{32}^{*}=\overline{\bar{\omega}}_{3}, k_{42}^{*}=\overline{\bar{\omega}}_{4}, k_{52}^{*}=\overline{\bar{\omega}}_{5}, k_{62}^{*}=\overline{\bar{\omega}}_{6}$, $k_{72}^{*}=\overline{\bar{\omega}}_{7}$ and $k_{82}^{*}=\overline{\bar{\omega}}_{8}$.

By exploiting (63), the inequality (B.1) becomes:

$\dot{V}_{3} \leq-k_{11} S_{\Omega}^{2}-\frac{\sigma_{\theta_{1}}}{2}\left\|\tilde{\theta}_{1}\right\|^{2}-\frac{\sigma_{k_{1}}}{2} \tilde{k}_{12}^{2}+\bar{\varepsilon}_{1}-S_{\varphi} \tilde{\theta}_{2}^{T} \psi_{2}\left(\bar{x}_{2}\right)+$

$k_{22}^{*} \bar{\varepsilon}_{i s d}-\tilde{k}_{22} S_{\varphi} \tanh \left(\frac{S_{\varphi}}{\varepsilon_{i s d}}\right)-k_{21} S_{\varphi}^{2}-S_{i s d 1} \tilde{\theta}_{3}^{T} \psi_{3}\left(\bar{x}_{3}\right)+$

$k_{32}^{*} \bar{\varepsilon}_{i s d 1}-\tilde{k}_{32} S_{i s d 1} \tanh \left(\frac{S_{i s d 1}}{\varepsilon_{i s d 1}}\right)-k_{31} S_{i s d 1}^{2}-$

$S_{i s q 1} \tilde{\theta}_{4}^{T} \psi_{4}\left(\bar{x}_{4}\right)+k_{42}^{*} \bar{\varepsilon}_{i s q 1}-\tilde{k}_{42} S_{i s q 1} \tanh \left(\frac{s_{i s q 1}}{\varepsilon_{i s q 1}}\right)-$ 
$k_{41} S_{i s q 1}^{2}-S_{i s o 1} \tilde{\theta}_{5}^{T} \psi_{5}\left(\bar{x}_{5}\right)+k_{52}^{*} \bar{\varepsilon}_{i s o 1}-$

$\tilde{k}_{52} S_{i s o 1} \tanh \left(\frac{S_{i s o 1}}{\varepsilon_{i s o 1}}\right)-k_{51} S_{i o s 1}^{2}-S_{i s d 2} \tilde{\theta}_{6}^{T} \psi_{6}\left(\bar{x}_{6}\right)+$

$k_{62}^{*} \bar{\varepsilon}_{i s d 2}-\tilde{k}_{62} S_{i s d 2} \tanh \left(\frac{s_{i s d 2}}{\varepsilon_{i s d 2}}\right)-k_{61} S_{i s d 2}^{2}-$

$S_{i s q 2} \tilde{\theta}_{7}^{T} \psi_{7}\left(\bar{x}_{7}\right)+k_{72}^{*} \bar{\varepsilon}_{i s q 2}-\tilde{k}_{72} S_{i s q 2} \tanh \left(\frac{s_{i s q 2}}{\varepsilon_{i s q 2}}\right)-$

$k_{71} S_{i s q 2}^{2}-S_{i s o 2} \tilde{\theta}_{8}^{T} \psi_{8}\left(\bar{x}_{8}\right)+k_{82}^{*} \bar{\varepsilon}_{i s o 2}$

$\tilde{k}_{82} S_{i s o 2} \tanh \left(\frac{S_{i S O 2}}{\varepsilon_{\text {iso } 2}}\right)-k_{81} S_{\text {iso } 2}^{2}$

where: $\bar{k}_{22}=k_{22}-k_{22}^{*}, \bar{k}_{32}=k_{32}-k_{32}^{*}, \bar{k}_{42}=k_{42}-k_{42}^{*}$, $\bar{k}_{52}=k_{52}-k_{52}^{*}, \bar{k}_{62}=k_{62}-k_{62}^{*}, \bar{k}_{72}=k_{72}-k_{72}^{*}, \bar{k}_{82}=$ $k_{82}-k_{82}^{*}, \bar{\varepsilon}_{i s d}=0.2785 \varepsilon_{i s d}, \bar{\varepsilon}_{i s d 1}=0.2785 \varepsilon_{i s d 1}, \bar{\varepsilon}_{i s q 1}=$ $0.2785 \varepsilon_{i s q 1}, \bar{\varepsilon}_{i s o 1}=0.2785 \varepsilon_{i s o 1}, \bar{\varepsilon}_{i s d 2}=0.2785 \varepsilon_{i s d 2}$, $\bar{\varepsilon}_{i s q 2}=0.2785 \varepsilon_{i s q 2}$ and $\bar{\varepsilon}_{i s o 2}=0.2785 \varepsilon_{i s o 2}$.

The derivation of $V_{4}$ gives:

$\dot{V}_{4} \leq-k_{11} S_{\Omega}^{2}-\frac{\sigma_{\theta_{1}}}{2}\left\|\tilde{\theta}_{1}\right\|^{2}-\frac{\sigma_{k_{1}}}{2} \tilde{k}_{12}^{2}+\bar{\varepsilon}_{1}-S_{\varphi} \tilde{\theta}_{2}^{T} \psi_{2}\left(\bar{x}_{2}\right)+$ $k_{22}^{*} \bar{\varepsilon}_{i s d}-\tilde{k}_{22} S_{\varphi} \tanh \left(\frac{S_{\varphi}}{\varepsilon_{i s d}}\right)-k_{21} S_{\varphi}^{2}-S_{i s d 1} \tilde{\theta}_{3}^{T} \psi_{3}\left(\bar{x}_{3}\right)+$ $k_{32}^{*} \bar{\varepsilon}_{i s d 1}-\tilde{k}_{32} S_{i s d 1} \tanh \left(\frac{s_{i s d 1}}{\varepsilon_{i s d 1}}\right)-k_{31} S_{i s d 1}^{2}-$ $S_{i s q 1} \tilde{\theta}_{4}^{T} \psi_{4}\left(\bar{x}_{4}\right)+k_{42}^{*} \bar{\varepsilon}_{i s q 1}-\tilde{k}_{42} S_{i s q 1} \tanh \left(\frac{s_{i s q 1}}{\varepsilon_{i s q 1}}\right)-$ $k_{41} S_{i s q 1}^{2}-S_{i s o 1} \tilde{\theta}_{5}^{T} \psi_{5}\left(\bar{x}_{5}\right)+k_{52}^{*} \bar{\varepsilon}_{i s o 1}-$ $\tilde{k}_{52} S_{i s o 1} \tanh \left(\frac{S_{i s o 1}}{\varepsilon_{i s o 1}}\right)-k_{51} S_{i o s 1}^{2}-S_{i s d 2} \tilde{\theta}_{6}^{T} \psi_{6}\left(\bar{x}_{6}\right)+$ $k_{62}^{*} \bar{\varepsilon}_{i s d 2}-\tilde{k}_{62} S_{i s d 2} \tanh \left(\frac{s_{i s d 2}}{\varepsilon_{i s d 2}}\right)-k_{61} S_{i s d 2}^{2}-$ $S_{i s q 2} \tilde{\theta}_{7}^{T} \psi_{7}\left(\bar{x}_{7}\right)+k_{72}^{*} \bar{\varepsilon}_{i s q 2}-\tilde{k}_{72} S_{i s q 2} \tanh \left(\frac{s_{i s q 2}}{\varepsilon_{i s q 2}}\right)-$ $k_{71} S_{i s q 2}^{2}-S_{i s o 2} \tilde{\theta}_{8}^{T} \psi_{8}\left(\bar{x}_{8}\right)+k_{82}^{*} \bar{\varepsilon}_{i s o 2}-$ $\tilde{k}_{82} S_{\text {iso } 2} \tanh \left(\frac{S_{\text {iso } 2}}{\varepsilon_{\text {iso } 2}}\right)-k_{82} S_{\text {iso } 2}^{2}+\frac{1}{2 \gamma_{\theta_{2}}} \tilde{\theta}_{2}^{T} \dot{\theta}_{2}+\frac{1}{2 \gamma_{k_{2}}} \tilde{k}_{22} \dot{k}_{22}+$ $\frac{1}{2 \gamma_{\theta_{3}}} \tilde{\theta}_{3}^{T} \dot{\theta}_{3}+\frac{1}{2 \gamma_{k_{3}}} \tilde{k}_{32} \dot{k}_{32}+\frac{1}{2 \gamma_{\theta_{4}}} \tilde{\theta}_{4}^{T} \dot{\theta}_{4}+\frac{1}{2 \gamma_{k_{4}}} \tilde{k}_{42} \dot{k}_{42}+$ $\frac{1}{2 \gamma_{\theta_{5}}} \tilde{\theta}_{5}^{T} \dot{\theta}_{5}+\frac{1}{2 \gamma_{k_{5}}} \tilde{k}_{52} \dot{k}_{52}+\frac{1}{2 \gamma_{\theta_{6}}} \tilde{\theta}_{6}^{T} \dot{\theta}_{6}+\frac{1}{2 \gamma_{k_{6}}} \tilde{k}_{62} \dot{k}_{62}+$ $\frac{1}{2 \gamma_{\theta_{7}}} \tilde{\theta}_{7}^{T} \dot{\theta}_{7}+\frac{1}{2 \gamma_{k_{7}}} \tilde{k}_{72} \dot{k}_{72}+\frac{1}{2 \gamma_{\theta_{8}}} \tilde{\theta}_{8}^{T} \dot{\theta}_{8}+\frac{1}{2 \gamma_{k_{8}}} \tilde{k}_{82} \dot{k}_{82}$

By using (63), we obtain:

$\dot{V}_{4} \leq-k_{11} S_{\Omega}^{2}-\frac{\sigma_{\theta_{1}}}{2}\left\|\tilde{\theta}_{1}\right\|^{2}-\frac{\sigma_{k_{1}}}{2} \tilde{k}_{12}^{2}-k_{21} S_{\varphi}^{2}-\frac{\sigma_{\theta_{2}}}{2}\left\|\tilde{\theta}_{2}\right\|^{2}-$ $\frac{\sigma_{k_{2}}}{2} \tilde{k}_{22}^{2}-k_{31} S_{i s d 1}^{2}-\frac{\sigma_{\theta_{3}}}{2}\left\|\tilde{\theta}_{3}\right\|^{2}-\frac{\sigma_{k_{3}}}{2} \tilde{k}_{32}^{2}-k_{41} S_{i s q 1}^{2}-$ $\frac{\sigma_{\theta_{4}}}{2}\left\|\tilde{\theta}_{4}\right\|^{2}-\frac{\sigma_{k_{4}}}{2} \tilde{k}_{42}^{2}-k_{51} S_{i s o 1}^{2}-\frac{\sigma_{\theta_{5}}}{2}\left\|\tilde{\theta}_{5}\right\|^{2}-\frac{\sigma_{k_{5}}}{2} \tilde{k}_{52}^{2}-$ $k_{61} S_{i s d 2}^{2}-\frac{\sigma_{\theta_{6}}}{2}\left\|\tilde{\theta}_{6}\right\|^{2}-\frac{\sigma_{k_{6}}}{2} \tilde{k}_{62}^{2}-k_{71} S_{i s q 2}^{2}-\frac{\sigma_{\theta_{7}}}{2}\left\|\tilde{\theta}_{7}\right\|^{2}-$ $\frac{\sigma_{k_{7}}}{2} \tilde{k}_{72}^{2}-k_{81} S_{i s o 2}^{2}-\frac{\sigma_{\theta_{8}}}{2}\left\|\tilde{\theta}_{8}\right\|^{2}-\frac{\sigma_{k_{8}}}{2} \tilde{k}_{82}^{2}+\varepsilon_{1}+\varepsilon_{2}+\varepsilon_{3}+$ $\varepsilon_{4}+\varepsilon_{5}+\varepsilon_{6}+\varepsilon_{7}+\varepsilon_{8}$

where:

$$
\begin{aligned}
& \bar{\varepsilon}_{2}=k_{22}^{*} \bar{\varepsilon}_{i s d}+\frac{\sigma_{\theta_{2}}}{2}\left\|\theta_{2}^{*}\right\|^{2}+\frac{\sigma_{k_{2}}}{2} k_{22}^{* 2}, \\
& \bar{\varepsilon}_{3}=k_{32}^{*} \bar{\varepsilon}_{i s d 1}+\frac{\sigma_{\theta_{3}}}{2}\left\|\theta_{3}^{*}\right\|^{2}+\frac{\sigma_{k_{3}}}{2} k_{32}^{* 2}, \\
& \bar{\varepsilon}_{4}=k_{42}^{*} \bar{\varepsilon}_{i s q 1}+\frac{\sigma_{\theta_{4}}}{2}\left\|\theta_{4}^{*}\right\|^{2}+\frac{\sigma_{k_{4}}}{2} k_{42}^{* 2}, \\
& \bar{\varepsilon}_{5}=k_{52}^{*} \bar{\varepsilon}_{i s o 1}+\frac{\sigma_{\theta_{5}}}{2}\left\|\theta_{5}^{*}\right\|^{2}+\frac{\sigma_{k_{5}}}{2} k_{52}^{* 2}, \\
& \bar{\varepsilon}_{6}=k_{62}^{*} \bar{\varepsilon}_{i s d 2}+\frac{\sigma_{\theta_{6}}}{2}\left\|\theta_{6}^{*}\right\|^{2}+\frac{\sigma_{k_{6}}}{2} k_{62}^{* 2}, \\
& \bar{\varepsilon}_{7}=k_{72}^{*} \bar{\varepsilon}_{i s q 2}+\frac{\sigma_{\theta_{7}}}{2}\left\|\theta_{7}^{*}\right\|^{2}+\frac{\sigma_{k_{7}}}{2} k_{72}^{* 2}, \\
& \bar{\varepsilon}_{8}=k_{82}^{*} \bar{\varepsilon}_{i s o 2}+\frac{\sigma_{\theta_{8}}}{2}\left\|\theta_{8}^{*}\right\|^{2}+\frac{\sigma_{k_{8}}}{2} k_{82}^{* 2} .
\end{aligned}
$$

